Worker-polymorphism accounts for very little of the division of labour in ants, although the soldier reacts differently to stimuli from its fellow workers.

The mechanism of the division of labour depends on the reaction of the individual ant, and it is of considerable interest that in the ants the factors of individual variation in behaviour and social integra. tion are complementary and not in conflict.

75 Gloucester Place,

Derek Wragge Morley

London, W.1.

${ }^{1}$ Schneirla, T. C., J. Comp. Psychol., 15, 243 (1933); 17, 303 (1934); 32. 41 (19+1); 35, 149 (1943) : J.' New York Ent. Soc., 52, 153 (1944) and other papers. Chen, S. C., Physiol. Zool., 10, 420, $437(1038)$.

Wragge Morley, B. D., in the press.

\section{Atmospheric Pressure Changes}

THE pressure in the atmosphere at any point is determined by the weight of air above that point, so that pressure changes depend on the three-dimensional field of motion. The relation is normally expressed by the pressure-tendency equation :

$$
\frac{\partial p}{\partial t}=-\int_{z}^{\infty} g \operatorname{div}(p v) d z
$$

where $\frac{\partial p}{\partial t}$ is the rate of change of pressure $p$ at height $z, g$ is the acceleration due to gravity, $\rho$ the density, and $\mathbf{v}$ the wind vector.

It is customary to examine the pressure tendencies associated with certain theoretical approximations to the wind. The large divergence of the geostropic wind $\mathbf{J}$ when the latter has a north or south component has recently led Jeffreys ${ }^{1}$ to recall the paradox that storms should move with a velocity com. parable with that of sound unless they have a special, but unknown, kind of structure.

This is not the case, since there is in the atmosphere a natural brake mechanism which forces all pressure systems to move with relatively slow speeds. A good approximation to the wind is afforded by the gradient wind $\mathbf{G}$, defined with its magnitude $G$ by the equation

$$
\mathbf{G}\left(1-\frac{G x}{\lambda}\right)=\mathbf{J} \text {, }
$$

where $x$ is the curvature of the trajectory (positive in anticyclonic motion) and $\lambda=2 \omega \sin \varphi, \omega$ being the earth's angular velocity and $\varphi$ the latitude. Making use of an expression due to Matthewman', the rate of change of pressure $p$ associated with the gradient approximation is, very closely,

$$
\frac{\partial p}{\partial t}=-\int_{0}^{p} \frac{1}{\lambda}\left\{x G \frac{\partial G}{\partial s}+G^{2} \frac{\partial x}{\partial s}-\frac{2 \omega \cos \varphi}{r} v_{\theta}\right\} d p,
$$

where $\frac{\partial}{\partial s}$ denotes space differentiation along the stream-line and $v_{G}$ is the south-north component of G. $r$ is the distance from the centre of the earth.

Each term on the right-hand side is potentially very large, and some further mechanism is required to explain why the observed pressure changes are invariably so small. In a non-steady pressure field, the trajectories of air particles differ from the instantaneous stream-lines. In order to show the mechanism of control, it is sufficient to represent this difference by

$$
G\left(x-x_{8}\right)=C x_{8} \sin \theta
$$

for a pressure system moving eastwards at speed $C$ without distortion. The suffix $s$ relates to the stream. $\operatorname{lin} \theta$, and $\theta$ is the angle from which the gradient wind blows, measured clockwise from north.

The tendency equation then takes the form

$$
\begin{aligned}
\frac{\partial p}{\partial t}=-\frac{1}{\lambda} \int_{0}^{p}\left[G \varkappa_{s} \frac{\partial G}{\partial s}+G(G+C \sin \theta)\right. & \\
& \left.\frac{\partial \varkappa_{s}}{\partial s}-C \varkappa_{s}{ }^{2} v_{G}-\frac{2 \omega \cos \varphi}{r} v_{G}\right] d p .
\end{aligned}
$$

Now $C$ is dependent on the pressure tendency itself, being, in fact, proportional to its local value. $\frac{\partial p}{\partial t}$ is, therefore, implicit on the right-hand side of the equation as well as explicit on the left, and one may effectively transpose the terms containing $C$ by writing

$F \frac{\partial p}{\partial t}=-\frac{1}{\lambda} \int_{0}^{p}\left(G \varkappa_{s} \frac{\partial G}{\partial s}+G^{2} \frac{\partial \varkappa_{s}}{\partial s}-\frac{2 \omega \cos \varphi}{r} v_{\theta}\right) d p$

It is possible to assess the magnitude of all terms, including $F$, from synoptic charts of the free atmosphere. Alternatively, one may carry the theoretical work a stage further by examining the typical upper pressure distributions associated with the commonly observed surface pressure systems. A more detailed account of the theoretical work will appear elsewhere, by courtesy of the Director of the Meteorological Office, London.

The equation has been applied to schematic models of warm-sector depressions, cold depressions, warm anti-cyclones, cold high-pressure ridges, developing secondary depressions and frontal waves, and to systems of straight north-south isobars. In each case $F$ may be represented explicitly in terms of the (vertically integrated) parameters of the pressure and stream-line fields. Its value is normally between 10 and 50 in middle latitudes, though it can be even higher : it may therefore be regarded as a control factor, and only a fraction of the large pressure tendencies suggested by the terms on the right-hand side of the above equations can in practice ever be realized.

The braking mechanism in the movement of pressure systems thus lies in the distortion of the trajectory which the movement itself creates. The normal movement of systems can be explained on the basis of the gradient wind approximation. (A discussion of this approximation is appended to the full paper.) By a refinement of the theory it is further possible to discriminate between fast- and slow-moving systems, and to show how the method is capable of yielding information on the develop. ment as well as the translation of the pressure system. Since only a contribution of the order of $25 \times \frac{\partial p}{\partial t}$ need be sought in examining the field of divergence, a much more hopeful outlook on the synoptic problem of pressure changes should emerge.

Whitecroft, C. H. B. Priestley

Little Chalfont,

Amersham.

Nov. 9.

'Jeffreys, H., Quart. J. Roy. Met. Soc., 117 (Jan. 1946).

Matthewman, A. G., Phil. Mag., in the press. 